PROCEEDINGS OF THE

AMERICAN MATHEMATICAL SOCIETY

Volume 136, Number 7, July 2008, Pages 2333-2341

S 0002-9939(08)09240-X

Article electronically published on March 13, 2008

\title{
ON ENDOMORPHISM RINGS OF LOCAL COHOMOLOGY MODULES
}

\author{
M. HELLUS AND J. STÜCKRAD
}

(Communicated by Bernd Ulrich)

\begin{abstract}
Let $R$ be a local complete ring. For an $R$-module $M$ the canonical ring map $R \rightarrow \operatorname{End}_{R}(M)$ is in general neither injective nor surjective; we show that it is bijective for every local cohomology module $M:=H_{I}^{h}(R)$ if $H_{I}^{l}(R)=0$ for every $l \neq h(=\operatorname{height}(I))(I$ an ideal of $R)$; furthermore the same holds for the Matlis dual of such a module. As an application we prove new criteria for an ideal to be a set-theoretic complete intersection.
\end{abstract}

\section{INTRODUCTION}

For an ideal $I$ of a local ring $(R, m)$ we denote the $n$-th local cohomology functor with support in $I$ by $H_{I}^{n}$ and the (contravariant) Matlis dual functor by $D$; i.e., $D(M)=\operatorname{Hom}_{R}(M, E)$ for any $R$-module $M$, where $E:=E_{R}(R / M)$ is a fixed $R$-injective hull of the residue field $R / m$.

Let $R$ be a (always commutative, unitary) ring and $M$ an $R$-module. Consider the canonical map $\mu_{M}: R \rightarrow \operatorname{End}_{R}(M)$ that maps $r \in R$ to multiplication by $r$ on $M$; it is a homomorphism of (associative) $R$-algebras. In general, $\mu_{M}$ is neither injective nor surjective. In section 2 we will show that, if $R$ is Noetherian local complete and $I$ an ideal of $R$ such that $H_{I}^{l}(R)=0$ for every $l \neq h(=\operatorname{height}(I))$, then $\mu_{H_{I}^{h}(R)}$ is bijective. In particular, the endomorphism ring of the $R$-module $H_{I}^{h}(R)$ is commutative and $\operatorname{Ann}_{R}\left(H_{I}^{h}(R)\right)=0$.

The proof of this result uses a generalization of Theorem 3.2 from [11, which says that, for a special class of Noetherian local complete rings $R$, it is true that $D\left(H_{I}^{h}\left(D\left(H_{I}^{h}(R)\right)\right)\right)$ is either zero or isomorphic to $R$ if $H_{I}^{l}(R)=0$ for every $l>$ $h=$ height $(I)$; the generalization is due to Khashyarmanesh ([14, Corollary 2.6]) and says that $D\left(H_{I}^{h}\left(D\left(H_{I}^{h}(R)\right)\right)\right) \cong R$ for every Noetherian local complete ring and every ideal $I \subseteq R$ such that $H_{I}^{l}(R)=0$ for $l \neq h=$ height $(I)$.

We also show in section 2 that $\mu_{D\left(H_{I}^{h}(R)\right)}$ is an isomorphism if $H_{I}^{l}(R)=0$ for every $l \neq h$.

Recently there was some work on Matlis duals of local cohomology modules (e.g. 6. 7, 10, 11, 12]). In [10, Corollary 1.1.4] the following was proved: If, for some $h \in \mathbb{N}, H_{I}^{l}(R)=0$ for all $l>h$ and $\underline{x}=x_{1}, \ldots, x_{h} \in I$ is an $R$-regular sequence,

Received by the editors February 21, 2007, and, in revised form, April 19, 2007.

2000 Mathematics Subject Classification. Primary 13C40; Secondary $13 \mathrm{C} 05$.

Key words and phrases. Local cohomology, endomorphism ring, Matlis dual, complete intersection.

(C)2008 American Mathematical Society Reverts to public domain 28 years from publication 
then one has the following equivalence:

$$
\sqrt{I}=\sqrt{\underline{x} R} \Longleftrightarrow \underline{x} \text { is a } D\left(H_{I}^{h}(R)\right) \text { regular sequence. }
$$

In section 3 we extend this equivalence:

Theorem. Let $(R, m)$ be a Noetherian local complete ring and $I$ an ideal of $R$ such that $H_{I}^{l}(R)=0$ for every $l>h:=\operatorname{height}(I) \geq 1$; let $\underline{x}=x_{1}, \ldots, x_{h} \in I$ be an $R$-regular sequence. Set $D:=D\left(H_{I}^{h}(R)\right)$. The following statements are equivalent:

(i) $\sqrt{I}=\sqrt{\underline{x} R}$; in particular, $I$ is a set-theoretic complete intersection.

(ii) $\underline{x}$ is a $D$-regular sequence.

(iii) The canonical map $D / \underline{x} D \rightarrow H_{\underline{x} R}^{h}(D)$ (coming from $H_{\underline{x} R}^{h}(D)=\underline{\lim }_{\longrightarrow \in \mathbb{N}} D$ / $\left.\underline{x}^{l} D\right)$ is injective.

(iv) The canonical map $\left\{r \in R \mid \forall_{l \in \mathbb{N}} \exists_{s \in \mathbb{N}} r \cdot I^{s} \subseteq \underline{x}^{l} R\right\} \rightarrow \Gamma_{I}(R / \underline{x} R)$ is surjective.

The equivalence of (ii) and (iii) is inspired by a result of Marley and Rogers (18, Prop. 2.3], which is a version of (ii) $\Longleftrightarrow$ (iii) for (arbitrary) finitely generated modules $D$.

\section{ENDOMORPHISM RINGS}

Definition 2.1. (i) Let $R$ be a ring and $M$ an $R$-module. The map

$$
R \rightarrow \operatorname{End}_{R}(M), \quad r \mapsto \text { multiplication by } r \text { on } M
$$

is an $R$-algebra homomorphism and will be denoted by $\mu_{M}$.

(ii) Let $R$ be a local ring and $M$ an $R$-module. $M$ has a canonical embedding

$$
M \rightarrow D(D(M))=D^{2}(M), \quad m \mapsto(\varphi \mapsto \varphi(m))
$$

into its bidual; this map will be denoted by $\iota_{M}$. We will consider $M$ as a submodule of $D^{2}(M)$ via $\iota_{M}$.

(iii) Let $(R, m)$ be a Noetherian local ring and $\underline{x}=x_{1}, \ldots, x_{h}$ a sequence of elements of $R$. For every $R$-module $M$ there is a canonical map

$$
M / \underline{x} M \stackrel{\iota_{M} \underline{x}}{\rightarrow} H_{\underline{x} R}^{h}(M)
$$

(coming from the description $H_{\underline{x} R}^{h}(M)=\underline{\lim }_{l \in \mathbb{N}} M /\left(x_{1}^{l}, \ldots, x_{h}^{l}\right) M$, where the transition maps are induced by multiplication by $\left.x_{1} \cdots x_{h}\right)$.

Theorem 2.2. Let $(R, m)$ be a Noetherian local complete ring and I an ideal of $R$ such that $H_{I}^{l}(R)=0$ for every $l \neq h$ ( $h$ is then necessarily the height of $I$ ). Set $H:=H_{I}^{h}(R)$.

(i) $\operatorname{Hom}_{R}\left(H, \iota_{H}\right): \operatorname{End}_{R}(H) \rightarrow \operatorname{Hom}_{R}\left(H, D^{2}(H)\right)$ is an isomorphism.

(ii) There is a canonical isomorphism

$$
\gamma_{H}: \operatorname{Hom}_{R}\left(H, D^{2}(H)\right) \rightarrow D\left(H_{I}^{h}(D(H))\right) .
$$

(iii) $\mu_{H}: R \rightarrow \operatorname{End}_{R}(H)$ is an isomorphism of $R$-algebras. Consequently there is a canonical isomorphism

$$
\gamma_{H} \circ \operatorname{Hom}_{R}\left(H, \iota_{H}\right) \circ \mu_{H}: R \rightarrow D\left(H_{I}^{h}(D(H))\right) .
$$

Proof. (i) It is clear that $\operatorname{Hom}_{R}\left(H, \iota_{H}\right)$ is injective. To show surjectivity, let $\varphi \in \operatorname{Hom}_{R}\left(H, D^{2}(H)\right)$ be arbitrary; let $x \in H$ be arbitrary and $n \in \mathbb{N}$ such that $I^{n} \cdot x=0$. This implies $I^{n} \cdot \varphi(x)=0$; i.e.

$$
\varphi(x) \in\left(0:_{D^{2}(H)} I^{n}\right)=D^{2}\left(\left(0:_{H} I^{n}\right)\right)=\left(0:_{H} I^{n}\right) \subseteq H
$$


(the first equality follows from exactness of $D$; for the second equality we remark that $\left(0:_{H} I^{n}\right)$ is finitely generated, as the spectral sequence

$$
E_{2}^{p, q}=\operatorname{Ext}_{R}^{p}\left(R / I^{n}, H_{I}^{q}(R)\right) \Rightarrow \operatorname{Ext}_{R}^{p+q}\left(R / I^{n}, R\right)
$$

shows $\left.\left(0:_{H} I^{n}\right)=\operatorname{Ext}_{R}^{h}\left(R / I^{n}, R\right)\right)$. This means that the image of $\varphi$ is contained in $H \subseteq D^{2}(H)$, which was precisely what we had to show.

(ii) Hom-Tensor adjointness shows

$$
\operatorname{Hom}_{R}\left(H, D^{2}(H)\right)=D\left(H \otimes_{R} D(H)\right) .
$$

On the other hand, our hypotheses imply $H_{I}^{l}=0$ for every $l>h$; in particular, $H_{I}^{h}$ is right exact, we get

$$
H \otimes_{R} D(H)=H_{I}^{h}(R) \otimes_{R} D(H)=H_{I}^{h}(D(H))
$$

and statement (ii) is now clear.

(iii) [14, Corollary 2.6] implies that there exists an isomorphism of $R$-modules

$$
D\left(H_{I}^{h}(D(H))\right) \cong R .
$$

Therefore, (i) and (ii) show that the $R$-module $\operatorname{End}_{R}(H)$ is free of rank one. Fix any isomorphism $R \cong \operatorname{End}_{R}(H)$ and let $\psi \in \operatorname{End}_{R}(H)$ be the element corresponding to $1 \in R$. In particular there exists a (unique) $x \in R$ such that $\operatorname{id}_{H}=x \circ \psi$, where $x$ is multiplication by $x$ on $H$. This implies $\varphi=x \circ \psi \circ \varphi$ for every $\varphi \in \operatorname{End}_{R}(H)$; in particular, multiplication by $x$ is surjective on $\operatorname{End}_{R}(H) \cong R, x$ is a unit in $R$ and $\mu_{H}$ is bijective.

Remark 2.3. The hypothesis of the previous theorem is fulfilled if $I$ is perfect and $R$ is regular and has positive characteristic. This was shown in [19, Prop. 4.1].

Corollary 2.4. In the situation of Theorem 2.2 the endomorphism ring of $H_{I}^{h}(R)$ is canonically isomorphic to $R$; in particular, it is commutative and $\operatorname{Ann}_{R}\left(H_{I}^{h}(R)\right)=$ 0 holds.

Let $(R, m)$ be a Noetherian local ring and $M$ an $R$-module. Consider the sequence of $R$-modules

$$
R \rightarrow \operatorname{End}_{R}(D(M)) \rightarrow \operatorname{End}_{R}\left(D^{2}(M)\right) \rightarrow \operatorname{Hom}_{R}\left(M, D^{2}(M)\right),
$$

where the first map is $\mu_{D(M)}$, the second is given by $\alpha \mapsto D(\alpha)$ and the third is restriction to $M \subseteq D^{2}(M)$. The composition of the second and third is always injective:

Lemma 2.5. Let $(R, m)$ be a Noetherian local ring and $M$ an $R$-module. The R-linear map

$$
\operatorname{End}_{R}(D(M)) \rightarrow \operatorname{Hom}_{R}\left(M, D^{2}(M)\right), \quad \varphi \mapsto\left(m \mapsto(D(\varphi))\left(\iota_{M}(m)\right)\right)
$$

is injective.

Proof. This is straightforward: Let $\varphi$ be in the kernel of the above map; this means that for all $m \in M$ and for all $\psi \in D(M)$ one has $\varphi(\psi)(m)=0$, i.e. $\varphi=0$.

We apply this injectivity in the case where the local ring $(R, m)$ is complete, $M:=H:=H_{I}^{h}(R)$ and $I$ is an ideal of $R$ such that $H_{I}^{l}(R)=0$ for every $l \neq h$; we 
get $R$-linear maps

$$
\begin{array}{rll}
R \rightarrow \operatorname{End}_{R}(D(H)) \rightarrow \operatorname{End}_{R}\left(D^{2}(H)\right) & \rightarrow & \operatorname{Hom}_{R}\left(H, D^{2}(H)\right) \\
& \stackrel{2.2}{=} \text { i) } & \operatorname{End}_{R}(H) \\
\stackrel{2.2(i i i)}{=} & R .
\end{array}
$$

The composition of all these maps is clearly $\operatorname{id}_{R}$. Thus, the injectivity statement from Lemma 2.5 shows:

Theorem 2.6. Let $(R, m)$ be a Noetherian local complete ring and $I$ an ideal of $R$ such that $H_{I}^{l}(R)=0$ for every $l \neq h$. Then the canonical map

$$
\mu_{D\left(H_{I}^{h}(R)\right)}: R \rightarrow \operatorname{End}_{R}\left(D\left(H_{I}^{h}(R)\right)\right)
$$

is an isomorphism of $R$-algebras.

\section{Complete intersections And local COHOMOlogy}

We need a couple of lemmata and remarks before we can prove Theorem 3.7 . which is the main result of this section:

Remark 3.1. Let $(R, m)$ be a Noetherian local ring, $I$ an ideal of $R$ and $M$ an $R$-module such that

$$
\operatorname{Supp}_{R}(M) \subseteq V(I)
$$

(where $V(I)=\{p \in \operatorname{Spec}(R) \mid p \supseteq I\})$. Let ^ denote $I$-adic completion. Then the natural map

$$
D(M) \rightarrow \widehat{D(M)}
$$

is an isomorphism; in particular, $\bigcap_{l \in \mathbb{N}} I^{l} \cdot D(M)=0$.

Proof. We have to show that the canonical map

$$
D(M) \rightarrow \lim _{i \in \mathbb{N}}\left(D(M) / I^{l} D(M)\right)
$$

is bijective, but one has

$$
\begin{aligned}
D(M) & =D\left(\Gamma_{I}(M)\right) \\
& =D(\underbrace{\lim }_{l \in \mathbb{N}} \operatorname{Hom}_{R}\left(R / I^{l}, M\right)) \\
& =\varliminf_{i \in \mathbb{N}} D\left(\operatorname{Hom}_{R}\left(R / I^{l}, M\right)\right) \\
& =\varliminf_{i \in \mathbb{N}} D(M) / I^{l} D(M),
\end{aligned}
$$

and it is easy to see that this is the canonical map $D(M) \rightarrow \widehat{D(M)}$.

Let $(R, m)$ be a Noetherian local ring and $\underline{x}=x_{1}, \ldots, x_{h}$ a sequence of elements of $R$. Marley and Rogers have shown ([18, Proposition 2.3]) that, for finitely generated $M, \iota_{M, \underline{x}}$ is injective iff $\underline{x}$ is an $M$-regular sequence. In this context, note that the proof of the following lemma is strongly based on their proof; our additional ingredient is Remark 3.1 
Lemma 3.2. Let $(R, m)$ be a Noetherian local ring, $I$ an ideal of $R, n, h \in \mathbb{N}$, $\underline{x}=x_{1}, \ldots, x_{h} \in I$ an arbitrary sequence and $N$ an $R$-module. Set $H:=H_{I}^{n}(N)$ and $D:=D(H)$. The following two statements are equivalent:

(i) For every $i=1, \ldots, h$, multiplication by $x_{i}$ on $D /\left(x_{1}, \ldots, x_{i-1}\right) D$ is injective (i.e., $\underline{x}$ is a D-quasiregular sequence).

(ii) $D / \underline{x} D \stackrel{\iota_{D, \underline{x}}}{\rightarrow} H_{\underline{x} R}^{h}(D)$ is injective.

Proof. (i) $\Rightarrow$ (ii): The finite case is well known; [20, Prop. 5.2.1] is a reference for the general case (note that (ii) holds trivially if $D / \underline{x} D=0$ ).

(ii) $\Rightarrow$ (i): By induction on $h: h=1$ : Set $x=x_{1}$ and let $\alpha \in D$ be such that $x \alpha=0$. We have to show $\alpha=0$. $\alpha$ represents an element of $\operatorname{ker}\left(\iota_{D, \underline{x}}\right)$; therefore, by assumption, $\alpha \in x D$. Choose $\alpha_{1} \in D$ such that $\alpha=x \alpha_{1}$. We conclude $x^{2} \alpha_{1}=0$. Again, $\alpha_{1}$ represents an element of $\operatorname{ker}\left(\iota_{D, \underline{x}}\right)$ and so there exists $\alpha_{2} \in D$ such that $\alpha_{1}=x \alpha_{2}$. Continuing in this way, we get

$$
\alpha \in \bigcap_{k \in \mathbb{N}} x^{k} D
$$

and then $\alpha=0$, by Remark 3.1, $h>1$ : First of all we prove injectivity of

$$
D /\left(x_{1}, \ldots, x_{h-1}\right) D \stackrel{\iota_{D, x_{1}, \ldots, x_{h-1}}}{\rightarrow} H_{\left(x_{1}, \ldots, x_{h-1}\right) R}^{h-1}(D) ;
$$

to do so, let $\alpha \in \operatorname{ker}\left(\iota_{D, x_{1}, \ldots, x_{h-1}}\right)$ be arbitrary. We show $\alpha \in\left(x_{1}, \ldots, x_{h-1}\right) D+x_{h}^{k} D$ for every $k \in \mathbb{N}$ by induction on $k: k=0$ is trivial, we assume $k>0$ and we write $\alpha=\omega+x_{h}^{k} \beta$ for some $\omega \in\left(x_{1}, \ldots, x_{h-1}\right) D, \beta \in D$. By our choice of $\alpha$ there exists $t \in \mathbb{N}$ such that

$$
\left(x_{1} \cdots x_{h-1}\right)^{t} x_{h}^{k} \beta \in\left(x_{1}^{t+1}, \ldots, x_{h-1}^{t+1}\right) D
$$

and hence

$$
\left(x_{1} \cdots x_{h}\right)^{t+k} \beta \in\left(x_{1}^{t+k+1}, \ldots, x_{h-1}^{t+k+1}\right) D .
$$

But $\iota_{D, \underline{x}}$ is injective; we conclude $\beta \in\left(x_{1}, \ldots, x_{h}\right) D$ and our induction on $k$ is finished:

$$
\alpha \in \bigcap_{k \in \mathbb{N}}\left(\left(x_{1}, \ldots, x_{h-1}\right) D+x_{h}^{k} D\right) .
$$

The $R$-module

$$
D /\left(x_{1}, \ldots, x_{h-1}\right) D=D\left(\operatorname{Hom}_{R}\left(R /\left(x_{1}, \ldots, x_{h-1}\right) R, H\right)\right)
$$

is $x_{h} R$-adically separated by Remark 3.1. This means

$$
\bigcap_{k \in \mathbb{N}}\left(\left(x_{1}, \ldots, x_{h-1}\right) D+x_{h}^{k} D\right)=\left(x_{1}, \ldots, x_{h-1}\right) D
$$

and the stated injectivity of $\iota_{D, x_{1}, \ldots, x_{h-1}}$ follows. The induction hypothesis shows that $x_{1}, \ldots, x_{h-1}$ is $D$-quasiregular; we have to show that multiplication by $x_{h}$ on $D /\left(x_{1}, \ldots, x_{h-1}\right) D$ is injective. Let $\alpha \in D$ be such that $x_{h} \alpha \in\left(x_{1}, \ldots, x_{h-1}\right) D$. We state

$$
\forall_{k \in \mathbb{N}} \alpha \in\left(x_{1}, \ldots, x_{h-1}\right) D+x_{h}^{k} D
$$

and prove this statement by induction on $k$. We may assume $k>0$ and write $\alpha=\omega+x_{h}^{k} \beta$ for some $\omega \in\left(x_{1}, \ldots, x_{h-1}\right) D, \beta \in D$. From $x_{h} \alpha=x_{h} \omega+x_{h}^{k+1} \beta$ we conclude $x_{h}^{k+1} \beta \in\left(x_{1}, \ldots, x_{h-1}\right) D$. Therefore,

$$
\left(x_{1}^{k+1} \cdots x_{h}^{k+1}\right) \beta \in\left(x_{1}^{k+2}, \ldots, x_{h-1}^{k+2}\right) D .
$$


But $\iota_{D, \underline{x}}$ is injective and so $\beta \in\left(x_{1}, \ldots, x_{h}\right) D$, and induction on $k$ is finished:

$$
\alpha \in \bigcap_{k \in \mathbb{N}}\left(\left(x_{1}, \ldots, x_{h-1}\right) D+x_{h} D\right) \stackrel{3.1}{=}\left(x_{1}, \ldots, x_{h-1}\right) D
$$

(note that the last equality has been explained above in a similar situation).

Let $(R, m)$ be a Noetherian local complete ring, $I$ an ideal of $R, h \in \mathbb{N}$; assume that $\underline{x}=x_{1}, \ldots, x_{h} \in I$ is an $R$-regular sequence. It follows from the Grothendieck spectral sequence belonging to the composed functors $\Gamma_{I} \circ \Gamma_{\underline{x} R}$ that

$$
H_{I}^{h}(R)=\Gamma_{I}\left(H_{\underline{x} R}^{h}(R)\right) \subseteq H_{\underline{x} R}^{h}(R) .
$$

By applying the functors $D, H_{\underline{x} R}^{h}$ and then $D$ again, we get a monomorphism (because $D$ is exact and $H_{\underline{x} R}^{h}$ is right exact)

$$
D\left(H_{\underline{x} R}^{h}\left(D\left(H_{I}^{h}(R)\right)\right)\right) \hookrightarrow D\left(H_{\underline{x} R}^{h}\left(D\left(H_{\underline{x} R}^{h}(R)\right)\right)\right) .
$$

Because of [14, Corollary 2.6], there is an isomorphism

$$
D\left(H_{\underline{x} R}^{h}\left(D\left(H_{\underline{x} R}^{h}(R)\right)\right)\right) \cong R .
$$

Clearly, this isomorphism is unique up to a unit of $R$ and so we may consider $D\left(H_{\underline{x} R}^{h}\left(D\left(H_{I}^{h}(R)\right)\right)\right)$ as an ideal of $R$ (alternatively we use Theorem 2.2 and have a canonical isomorphism $D\left(H_{\underline{x} R}^{h}\left(D\left(H_{\underline{x} R}^{h}(R)\right)\right)\right)=R$; the resulting ideal $J_{\underline{x}, I}$ is the same in both cases).

Definition 3.3. In the above situation, set

$$
J_{\underline{x}, I}:=D\left(H_{\underline{x} R}^{h}\left(D\left(H_{I}^{h}(R)\right)\right)\right)
$$

and consider $J_{\underline{x}, I}$ as an ideal of $R$.

Remark 3.4. Though the definition of $J_{\underline{x}, I}$ is quite abstract, it also has the following concrete description: Because of the right exactness of $H_{\underline{x} R}^{h}$,

$$
D\left(H_{\underline{x} R}^{h}\left(D\left(H_{I}^{h}(R)\right)\right)\right)=D\left(H_{\underline{x} R}^{h}(R) \otimes_{R} D\left(H_{I}^{h}(R)\right)\right)
$$

and by Hom-Tensor adjointness, the latter module is

$$
\operatorname{Hom}_{R}\left(H_{\underline{x} R}^{h}(R), D^{2}\left(H_{I}^{h}(R)\right)\right) \text {. }
$$

Now the arguments from the proof of Theorem 2.2 (i) show

$$
\operatorname{Hom}_{R}\left(H_{\underline{x} R}^{h}(R), D^{2}\left(H_{I}^{h}(R)\right)\right)=\operatorname{Hom}_{R}\left(H_{\underline{x} R}^{h}(R), H_{I}^{h}(R)\right) .
$$

But $H_{I}^{h}(R)=\Gamma_{I}\left(H_{\underline{x} R}^{h}(R)\right)$ and we get

$$
J_{\underline{x}, I}=\left\{\varphi \in \operatorname{End}_{R}\left(H_{\underline{x} R}^{h}(R)\right) \mid \operatorname{im}(\varphi) \subseteq \Gamma_{I}\left(H_{\underline{x} R}^{h}(R)\right)\right\} .
$$

We have $\operatorname{End}_{R}\left(H_{\underline{x} R}^{h}(R)\right) \stackrel{\text { can }}{=} R$ and thus

$$
J_{\underline{x}, I}=\left\{r \in R \mid r \cdot H_{\underline{x} R}^{h}(R) \subseteq \Gamma_{I}\left(H_{\underline{x} R}^{h}(R)\right) .\right.
$$

Using the description $H_{\underline{x} R}^{h}(R)=\bigcup_{l \in \mathbb{N}} R / \underline{x}^{l} R$ (where $\underline{x}^{l}=x_{1}^{l}, \ldots, x_{h}^{l}$ ), we conclude

$$
J_{\underline{x}, I}=\left\{r \in R \mid \forall_{l \in \mathbb{N}} \exists_{s \in \mathbb{N}} r \cdot I^{s} \subseteq \underline{x}^{l} R\right\}=\bigcap_{l \in \mathbb{N}}\left(\underline{x}^{l} R:\langle I\rangle\right) .
$$


Therefore, if we restrict the canonical map $R \rightarrow R / \underline{x} R$ to $J_{\underline{x}, I}$, we get a canonical map from $J_{\underline{x}, I}$ to $\Gamma_{I}(R / \underline{x} R)$ :

Definition 3.5. In the above situation, the canonical map

$$
J_{\underline{x}, I} \rightarrow \Gamma_{I}(R / \underline{x} R)
$$

is denoted by $j_{\underline{x}, I}$.

Remark 3.6. Let $(R, m)$ be a Noetherian local complete ring. Let $I$ be an ideal of $R, h \in \mathbb{N}$ and

$$
\underline{x}=x_{1}, \ldots, x_{h} \subseteq I
$$

an $R$-regular sequence. Then

$$
I \subseteq \sqrt{\underline{x} R+\operatorname{Ann}_{R}\left(J_{\underline{x}, I}\right)} .
$$

In particular, if $R$ is a domain and $\sqrt{\underline{x} R} \subsetneq \sqrt{I}$, then $J_{\underline{x}, R}=0$.

Proof. We use the description of $J_{\underline{x}, R}$ from Remark 3.4. For the first statement we have to show

$$
V\left(\operatorname{Ann}_{R}\left(J_{\underline{x}, I}\right)\right) \cap V(\underline{x} R) \subseteq V(I) ;
$$

i.e., for every $r \in J_{\underline{x}, R}$ we have to show

$$
V\left(\operatorname{Ann}_{R}(r)\right) \cap V(\underline{x} R) \subseteq V(I) .
$$

Let $r \in J_{\underline{x}, I}$ be arbitrary; by Remark $\underline{3.4}, J_{\underline{x}, I}=\left\{r \in R \mid \forall_{l \in \mathbb{N}} \exists_{s \in \mathbb{N}} r \cdot I^{s} \subseteq \underline{x}^{l} R\right\}$. For every $p \in V(\underline{x} R) \backslash V(I)$ we get

$$
r \cdot R_{p} \subseteq \bigcap_{l \in \mathbb{N}} \underline{x}^{l} R_{p} \subseteq \bigcap_{l \in \mathbb{N}} p^{l} R_{p}=0 ;
$$

i.e., $\operatorname{Ann}_{R}(r) \nsubseteq p$ and the first statement is proven. The second statement follows immediately from the first.

Theorem 3.7. Let $(R, m)$ be a Noetherian local complete ring and $I$ an ideal of $R$ such that $H_{I}^{l}(R)=0$ for every $l>h:=\operatorname{height}(I) \geq 1$; let $\underline{x}=x_{1}, \ldots, x_{h} \in I$ be an $R$-regular sequence (clearly, this implies $H_{I}^{l}(R)=0$ for every $l \neq h$ ). Set $D:=D\left(H_{I}^{h}(R)\right)$. The following statements are equivalent:

(i) $\sqrt{I}=\sqrt{\underline{x} R}$; in particular, $I$ is a set-theoretic complete intersection.

(ii) $\underline{x}$ is a D-regular sequence.

(iii) $D / \underline{x} D \stackrel{\iota^{D, \underline{x}}}{\rightarrow} H_{\underline{x} R}^{h}(D)$ is injective.

(iv) $j_{\underline{x}, I}$ is surjective.

(v) $J_{\underline{x}, I}=R$.

Proof. (i) $\Longleftrightarrow$ (ii) was shown (for more general $R$ ) in [10, Cor. 1.1.4].

(ii) $\Longleftrightarrow$ (iii) is a special case of Lemma $\underline{3.2}$ (note that $D / \underline{x} D=D\left(\operatorname{Hom}_{R}(R / \underline{x} R\right.$, $\left.\left.H_{I}^{h}(R)\right)\right) \neq 0$ ).

(iii) $\Longleftrightarrow$ (iv): By definition, $D\left(H_{\underline{x} R}^{h}(D)\right)=J_{\underline{x}, I}$. We have

$$
D(D / \underline{x} D)=D\left(D\left(\operatorname{Hom}_{R}\left(R / \underline{x} R, H_{I}^{h}(R)\right)\right)\right) .
$$


But $H_{I}^{h}(R)=\Gamma_{I}\left(H_{\underline{x} R}^{h}(R)\right)$ and, therefore,

$$
\begin{aligned}
\operatorname{Hom}_{R}\left(R / \underline{x} R, H_{I}^{h}(R)\right) & =\Gamma_{I}\left(\operatorname{Hom}_{R}\left(R / \underline{x}, H_{\underline{x} R}^{h}(R)\right)\right) \\
& =\Gamma_{I}\left(\operatorname{Ext}_{R}^{h}(R / \underline{x} R, R)\right) \\
& =\Gamma_{I}(R / \underline{x} R)
\end{aligned}
$$

(for the second and the third equality use the fact that $\underline{x}$ is an $R$-regular sequence). The latter module is finitely generated. We get

$$
D\left(D\left(\operatorname{Hom}_{R}\left(R / \underline{x} R, H_{I}^{h}(R)\right)\right)\right)=\Gamma_{I}(R / \underline{x} R) .
$$

Thus $D\left(\iota_{D, \underline{x}}\right)$ is a map $J_{\underline{x}, I} \rightarrow \Gamma_{I}(R / \underline{x} R)$; it is straightforward to see that it is in fact $j_{\underline{x}, I}$ (to do so one should start with the description $H_{\underline{x} R}^{h}(D)=\lim _{\longrightarrow \in \mathbb{N}}\left(D / \underline{x}^{l} D\right)$.

Question 3.8. In the situation of Definition 3.3 , when exactly is $J_{\underline{x}, I}=0$ ?

\section{REFERENCES}

[1] Bruns, W., and Herzog, J., Cohen-Macaulay Rings, Cambridge Univ. Press (1993). MR 1251956 (95h:13020)

[2] Bjork, J.-E., Rings of Differential Operators, North-Holland, Amsterdam (1979). MR.549189 (82g:32013)

[3] Brodmann, M. P., and Sharp, R. Y., Local Cohomology: An Algebraic Introduction with Geometric Applications, Cambridge Stud. Adv. Math. 60 (1998). MR.1613627(99h:13020)

[4] Bresinsky, H., Stückrad, J., and Renschuch, B., Mengentheoretisch vollständige Durchschnitte verschiedener rationaler Raumkurven im $\mathbf{P}^{3}$ über Körpern von Primzahlcharakteristik, Math. Nachr. 104 (1981), 147-169. MR657889 (84j:14051)

[5] Grothendieck, A., Local Cohomology, Lecture Notes in Math., No. 41, Springer-Verlag, Berlin-New York (1967). MR0224620 (37:219)

[6] Hellus, M., On the associated primes of Matlis duals of top local cohomology modules, Comm. Algebra 33 (2005), no. 11, 3997-4009. MR2183976 (2006h:13026)

[7] Hellus, M., Matlis duals of top local cohomology modules and the arithmetic rank of an ideal, Comm. Algebra 35 (2007), 1421-1432. MR2313677

[8] Hellus, M., Attached primes and Matlis duals of local cohomology modules, Arch. Math. (Basel) Archiv der Mathematik 89 (2007), 202-210. MR2352387

[9] Hellus, M., Local homology, Cohen-Macaulayness and Cohen-Macaulayfications, Algebra Colloquium 15:1 (2008), 63-68.

[10] Hellus, M., Local cohomology and Matlis duality, Habilitationsschrift, Leipzig (2006).

[11] Hellus, M., Finiteness properties of duals of local cohomology modules, Communications in Algebra 35 (2007), 3590-3602. MR2362672

[12] Hellus, M., and Stückrad, J., Matlis duals of top local cohomology modules, Proceedings of the AMS 136 (2008), 489-498. MR2358488

[13] Hellus, M., and Stückrad, J., Artinianness of local cohomology, submitted to Journal of Commutative Algebra.

[14] Khashyarmanesh, K., On the Matlis duals of local cohomology modules, Arch. Math. (Basel) 88 (2007), 413-418. MR2316886

[15] Lyubeznik, G., Finiteness properties of local cohomology modules (an application of $D$ modules to commutative algebra), Invent. Math. 113 (1993), 41-55. MR1223223 (94e:13032)

[16] Matlis, E., Injective modules over Noetherian rings, Pacific J. Math. 8 (1958), 511-528. MR0099360 (20:5800)

[17] Matsumura, H., Commutative Ring Theory, Cambridge Stud. Adv. Math. 8, Cambridge University Press, Cambridge (1986). MR879273 (88h:13001)

[18] Marley, T., and Rogers, M., Gorenstein rings and irreducible parameter ideals, arXiv:math.AC/0608661. 
[19] Peskine, C., and Szpiro, L., Dimension projective finie et cohomologie locale, Inst. Hautes Études Sci. Publ. Math. 42 (1973), 47-119. MR0374130(51:10330)

[20] Strooker, J. R., Homological Questions in Local Algebra, London Math. Soc. Lecture Note Ser. 145, Cambridge University Press, Cambridge (1990). MR.1074178 (91m:13013)

Universität Leipzig, Fakultät für Mathematik und Informatik, PF 1009 20, D-04009, LEIPZig, GERMANY

E-mail address: hellus@math.uni-leipzig.de

Universität Leipzig, Fakultät für Mathematik und Informatik, PF 1009 20, D-04009, LeipZig, Germany

E-mail address: stueckrad@math.uni-leipzig.de 